\title{
Der Begriff Entwicklung im Freiwilligendienst Weltwärts - Ansichten von Süd-Nord-Freiwilligen aus postkolonialer Perspektive
}

\author{
Lisa Bergmann \\ Referentin im Süd-Nord-Freiwilligenprogramm der Vereinten Ev. Mission \\ bergmann-I@vemission.org
}

\section{Zusammenfassung}

In einer groß angelegten Evaluation der Weltwärts Süd-Nord-Komponente im Jahr 2017 wurde die Frage aufgeworfen, was genau das Entwicklungspolitische an der Süd-NordKomponente sei. Bisher wurde die Thematik vielfältig diskutiert aber noch nicht in Gänze beantwortet. Der vorliegende Beitrag geht der Frage der Entwicklung nach und wird die Ansichten von Süd-Nord-Freiwilligen in den Mittelpunkt der Analyse stellen: Wie fassen sie „Entwicklung“ als Teil ihres Dienstes? Findet Entwicklung statt und wenn ja auf welchen Ebenen? Was für Änderungsprozesse können nachverfolgt werden?

Als theoretischer Hintergrund des Aufsatzes wird die postkoloniale Theorie gewählt, mit der die verschiedenen genannten Aspekte beleuchtet werden. Der Beitrag arbeitet heraus, dass der Freiwilligendienst vielschichtige Formen von Entwicklung anregt, welche sowohl Auswirkungen auf das Gastland Deutschland als auch das Heimatland haben können. Entwicklungen können ökonomischer Art sein, umfassen aber mehr noch ein Umdenken, eine Horizonterweiterung und gesellschaftliche Transformationsprozesse.

Schlagwörter: Weltwärts; postkolonial; Süd-Nord-Freiwilligendienst; Entwicklung; Entwicklungspolitik

\begin{abstract}
In major study regarding the Weltwärts South-North-component, which was published in 2017, the question was posed what the development political aspects of the South-North-component were. So far, this topic has been widely discussed, but could not be answered entirely. This paper is going to pick up the subject by focusing on South-North volunteers' perspectives on the topic: How do volunteers capture development as part of their service? Does development take place and, if so, on what levels? What kind of changing processes can be depicted? Postcolonial Theory will serve as theoretical background to analyze the various topics mentioned. The paper examines that the volunteer service fosters various kinds of development, which may have an effect on both the host country Germany as well as the respective home country. Development processes may be economical, but also encompass new perspectives, widening of horizons and societal transformation processes. Keywords: Weltwärts; postcolonialism; South-North-volunteering; development; development policies
\end{abstract}

\section{Einleitung}

Der Freiwilligendienst Weltwärts ist beim Bundesministerium für wirtschaftliche Zusammenarbeit und Entwicklung (BMZ) angesiedelt und somit Teil der 
deutschen Entwicklungspolitik. Was genau junge Freiwillige zur Entwicklungsarbeit beitragen können, ist eine Debatte, die schon bald nach Einführung des Programms 2008 aufgegriffen wurde (bspw. durch Kontzi 2011). Während zu Beginn Nord-Süd-Freiwillige noch als (Entwicklungs-)Helfer_innen konstruiert wurden, hat sich der Diskurs nun verschoben hin zu der Annahme, dass Freiwillige primär Lernende sind. Sie unterstützen während ihres Jahres entwicklungspolitische Projekte, stärken so Partnerschaften und engagieren sich nach ihrer Rückkehr in Deutschland (Polak/Guffler/Scheinert 2017: vii f.; Kontzi 2015).

Doch wie stellt sich die Situation innerhalb der Süd-Nord-Komponente dar? Nachdem sie im Jahr 2013 zunächst als Pilotprojekt initialisiert wurde, gab es eine groß angelegte Evaluation, deren Ergebnisse im Sommer 2017 veröffentlicht wurden. Der Evaluationsbericht wirft die Frage auf, was das Entwicklungspolitische am Freiwilligendienst Weltwärts sei (Scheller/Krämer/Hielscher 2017: viii; 37 ff.). Bisher hat sich keine Studie weitergehend mit diesem Thema befasst; Grundsätzlich gibt es wenige Untersuchungen, die sich mit dem Süd-Nord-Freiwilligendienst auseinandersetzen. Anzuführen sind die Studien von Repenning (2016) und Skoruppa (2016). Der vorliegende Aufsatz schließt diese Forschungslücke. Dabei sollen die Ideen und Ansichten der Süd-Nord-Freiwilligen selbst in den Mittelpunkt gestellt werden. Die Forschungsfrage lautet: Wie sehen Süd-Nord-Freiwillige die Frage der Entwicklung in ihrem entwicklungspolitischen Freiwilligendienst?

Der Beitrag stellt Teilergebnisse einer im Rahmen einer Masterarbeit durchgeführten Studie vor, basierend auf einem explorativ-induktiven Forschungsvorgehen. Den theoretischen Rahmen bildet die postkoloniale Theorie. Dieser interdisziplinäre Ansatz beschäftigt sich mit verschiedenen Auswirkungen der Kolonialgeschichte und analysiert unter anderem den modernen Entwicklungsdiskurs. Nach einer kurzen Einführung in die postkoloniale Theorie wird das methodische Vorgehen vorgestellt, um dann auf die Ergebnisse der Interviews einzugehen.

\section{Theoretischer Rahmen: Entwicklungszusammenarbeit im postkolonialen Kontext}

Den theoretischen Hintergrund der Fragestellung, wie Süd-Nord-Freiwillige Entwicklung im Rahmen ihres Dienstes sehen, bildet die postkoloniale Theorie. Diese beschäftigt sich mit den Nachwirkungen des Kolonialismus in seinen vielfältigen Facetten. (Ziai 2016 402; Kerner 2012: 9; Conrad 2012: 4). Diese Nachwirkungen betreffen die verschiedensten Felder des öffentlichen und privaten Lebens, sodass postkoloniale Studien von hoher Interdisziplinarität geprägt sind. Ziel ist es, zentrale koloniale Annahmen (wie z. B. des Rassismus) zu dekonstruieren und so zu überwinden (Castro Varela/Dhawan 2015: 17). Postkoloniale Theorien „,[erheben] den Anspruch [...], auf gesellschaftliche Missstände einschließlich ihrer Ursachen und Wirkungen aufmerksam zu machen und dadurch dazu beizutragen, diese 
Missstände zu beheben“ (Kerner 2012: 12). Die zentralsten Autor_innen sind Edward Said (1978), Gayatri Spivak (1988) und Homi K. Bhabha (1994). Auch Dipesh Chakrabarty (2002) ist an dieser Stelle zu nennen. Edward Said analysiert in seinem Buch „Orientalism“ (1978), wie der Orient im Westen von (vermeintlichen) Expert_innen konstruiert wurde. Dieses Konstrukt ist „das Andere“ als Gegenbild zum Selbst; das Gegensätzliche zum „aufgeklärten“, „entwickelten“ Europa. Die Autorin Gyatri Chakravorty Spivak schrieb 1988 ihren vielbeachteten Essay „Can the Subaltern Speak?“. In diesem knüpft sie inhaltlich an Said und Foucault an, beleuchtet das Thema der Postkolonialität aber von einer feministischeren und marxistischeren Perspektive. Sie geht dabei auf Subalterne, also marginalisierte Personen, die nicht oder kaum repräsentiert sind, ein. Häufig sind dies Frauen (Spivak 1988: 83). Homi K. Bhabha untersucht die Subjektbildung (Subjektivierung) innerhalb des kolonialen und postkolonialen Diskurses und fokussiert die Frage der Hybridität in seinen Schriften (Bhabha 1994). Chakrabarty arbeitet in seinen Studien unter anderem den Eurozentrismus vieler Konzepte und gängiger Wissensformen heraus und fordert, Europa zu provinzialisieren (Chakrabarty 2002: 283).

Auch die moderne Entwicklungszusammenarbeit ist Untersuchungsgegenstand postkolonialer Theorie. In diesem Kontext wird die Frage gestellt, wieso sich ehemalige Kolonien an den vom Westen gesetzten Normen von Staatlichkeit und ökonomischer Entwicklung orientieren sollten, wenn dies dazu führt, dass die eigene Lebensart ständig als ein Noch-Nicht-Erreichen des Ideals (Modernität und ökonomisches Wachstum) dargestellt wird (Kontzi 2015: 133). Hall (1994) formte mit Blick darauf den Begriff „des Westens und des Rests“. Daraus ergibt sich zum einen eine globale Hierarchie, an deren Spitze Länder mit hoher Wirtschaftsleistung stehen. Zum anderen umfasst sie eine pfadabhängige Entwicklungsidee. Dabei habe Entwicklung so abzulaufen und das gleiche Resultat zu erbringen, wie in Ländern des Globalen Nordens (Eckert 2006: 3).

Ferner fließen im Rahmen von Entwicklungszusammenarbeit große Summen vom Globalen Norden in den Süden. Monetäre Geldflüsse bedeuten jedoch Abhängigkeit und Macht (Müller/Ziai 2015: 9; glokal e. V.: 16). Auch wenn in der heutigen Zeit versucht wird, auf Augenhöhe zu arbeiten (die Änderung der Begrifflichkeit von Entwicklungshilfe in Entwicklungszusammenarbeit [EZ] ist eine Ausgestaltung dieser Tatsache), wird der EZ weiterhin Paternalismus vorgeworfen (Ziai 2016; Baaz 2005; glokal e. V. 2016)

Das Konzept des Süd-Nord-Freiwilligendienstes bricht in gewisser Weise mit den Annahmen klassischer Entwicklungszusammenarbeit. Weder Personal noch Expertise fließen in diesem Fall vom Norden in den Süden, sondern der Austausch funktioniert andersherum: vom Süden in den Norden. Die Macht des Geldes allerdings bleibt in Deutschland. Beim Studium der Zielsetzungen des Programms fällt 
auf, dass die Entwicklung der Heimatkulturen der Freiwilligen (nämlich als Multiplikator_innen in der Stärkung der Zivilgesellschaft) nur eines von sechs Zielen des BMZ hinsichtlich übergeordneter entwicklungspolitischer Wirkungen ist. Im Fokus steht die Stärkung der Partnerschaftsarbeit, Bewusstseinsaustausch oder die Stärkung der Zivilgesellschaft - in Deutschland wie im Heimatland (Scheller/ Krämer/Hielscher 2017).

Fraglich ist nun, wie die befragten Freiwilligen ihre Tätigkeit einordnen: Sehen sie ihre Tätigkeit als Entwicklungsarbeit? Welche Dimensionen von Entwicklung entwickeln sie? Diesen Fragen geht die anschließende Forschung nach.

\section{Methodik, Forschungsfeld und Rolle}

Der Fragestellung wird sich einem qualitativen Ansatz folgend induktiv genähert. Forschungsphilosophisch wird das Ziel verfolgt zu verstehen, „wie die soziale und politische Welt beschaffen ist und [...] wie wir Erkenntnisse über diese Welt in unserer Forschung erhalten können“ (Blatter/Langer/Wagemann 2017: 17). Dabei geht es darum, offen an einen Forschungsgegenstand heranzugehen und Lebenswelten „von innen hinaus“ zu verstehen (Flick/Kardoff/Steinke 2007: 14). Das Subjekt wird in den Mittelpunkt der Forschung gerückt und versucht, soziale Gegenstände zu erfassen (Reichertz 2014: 70; Heinze 2001: 14).

Meine Gesprächspartner_innen waren Süd-Nord-Freiwillige, die über ein Missionswerk in Deutschland am Weltwärts-Programm teilgenommen haben, der Großteil (13 Personen) war zum Zeitpunkt der Befragung circa ein halbes Jahr in Deutschland, zwei Interviewte hatten den Dienst bereits abgeschlossen. Insgesamt wurden also 15 narrativ geprägte Leitfrageninterviews geführt, davon sechs im persönlichen Gespräch und neun telefonisch. Die Interviewfragen umfassten verschiedenste Thematiken. Dieser Beitrag befasst sich insbesondere mit einem der in den Interviews aufgegriffenen Themen, nämlich dem der Entwicklung. Die entsprechende Interviewfrage lautete: „The program is financed by the German ministry of development. Do you see your work as development cooperation?" Einfluss fand aber auch die Frage, ob die Interviewten sich mit Rassismus konfrontiert sähen.

Die Auswertung der Gespräche folgte den Vorgaben der qualitativen Inhaltsanalyse nach Philipp Mayring (2015; 2002). Diese stellt ein „systematisches, regelgeleitetes Vorgehen“ (Mayring 2015: 50) dar, mit dessen Hilfe der Interviewkorpus ausgewertet werden kann. Hierfür wurden die transkribierten Interviews (1) paraphrasiert (zusammenfassen), (2) generalisiert (Abstraktionsniveau finden), (3) reduziert (unwichtiges streichen) und (4) nochmals reduziert, um Dinge zu bündeln (Mayring 2015: 72). Diese Schritte können auch mehrmals angewandt werden. Am Ende steht ein Kategoriensystem, das bei der Auswertung der Fragestellung hilft. 


\section{Ansichten von Süd-Nord-Freiwilligen zur Frage der Entwicklung}

In den Interviews zeigte sich, dass die Freiwilligen verschiedene Ideen zur Frage der Entwicklung im Rahmen ihres Dienstes haben. Diese sollen im Folgenden vorgestellt werden und dabei in die Themenfelder (1) persönliche Entwicklung, (2) Entwicklungstendenzen in der Heimat und (3) Helfen und Spenden aufgeteilt werden.

Eine interessante Erkenntnis der Interviews war, dass die Frage nach der eigenen Arbeit als Entwicklungszusammenarbeit in Deutschland einiges Nachdenken auslöste. Dieses Nachdenken spiegelt die Ergebnisse des Weltwärts Süd-Nord Evaluationsberichtes von 2017 wider (Scheller/Krämer/Hielscher 2017: xii). Das Entwicklungspolitische in der Süd-Nord-Komponente scheint sowohl für die Freiwilligen selbst als auch für die Autor_innen des Evaluationsberichts schwierig greifbar. Es wird deutlich, dass Entwicklung eine Reihe von Dimensionen hat und nicht nur aus dem Blickwinkel von rein ökonomischer Entwicklung gedacht werden sollte. Dies deckt sich mit den Ansätzen der post-development-Kritik, wie etwa von Escobar (1995).

\subsubsection{Persönliche Entwicklung}

Die meistgelieferte Interpretation des Dienstes war diejenige der persönlichen Entwicklung. Sie zieht sich durch alle Interviews und stellt das verbindende Element dar zwischen Weiterentwicklungen in Deutschland (bspw. in der Einsatzstelle) und in der Heimat. Exemplarisch hierfür sind die Aussagen einer Freiwilligen aus Indien:

But, for me, personally, I have grown strong in the last one year, I would say. [...] My viewpoint, my perspective has changed. I don't know [...] there is more confidence in terms of how I could create social changes. Maybe ... Maybe the development is myself.

Der Freiwilligendienst hat augenscheinlich eine höchst ermächtigende Wirkung auf die persönliche Entwicklung. Der Weltwärts-Evaluationsbericht bestätigt dies: „Die Süd-Nord-Freiwilligen haben sich in einem nonformalen Lern- und Bildungsprozess weiterentwickelt“ (Scheller/Krämer/Hielscher 2017: ix).

\subsubsection{Entwicklungstendenzen in Deutschland und in der Heimat}

Aus den Interviews wird deutlich, dass Freiwillige Inspiration und Motivation für Änderungen in der Heimat finden. Gleichzeitig versuchen sie, Klischees und Rassismen, die ihnen in Deutschland begegnen, aufzubrechen. 
Ein Freiwilliger aus Ghana fasst seine Interpretation von Entwicklung wie folgt zusammen:

Development touches with human lives. [...] I know that ever since I came here I made a difference in the life of someone. This is a way of development. And I think that this has a replicating effect on others. They would also go out and make an impression on someone else. And this causes a development. And also, you see, a development has something to do with a global view. A worldwide view. [...] I want to talk to the youth in the church and to talk about Germany, to talk about their culture, to talk about what this program is about and the impact I had and what are the changes that occurred when I was in Germany for one year. [...] It can impact into their lives, also.

Die Aussage zeigt einen globalen Ansatz von Entwicklung, der nur über gemeinsamen Austausch untereinander erreicht werden kann. Der Freiwillige selbst ist Multiplikator und sieht sich in der Position der Verantwortung, globale Entwicklung voranzutreiben.

Im letzten Kapitel wurde eine Freiwillige zitiert, die nicht nur sagt, dass sie selbst sich entwickelt habe („Maybe the development is myself.“), sondern auch, dass sie nun mehr Vertrauen habe, sozialen Wandel hervorzurufen: „There is more confidence in terms of how I could create social changes." An dieser Stelle wird das politische Potential des Freiwilligendienstes deutlich: Er ermöglicht es Menschen, politische Handlungsfähigkeit zu erproben und sich aktiv in gesellschaftliche Prozesse einzubringen und diese zu formen.

Auch bietet die Arbeit in Deutschland Inspiration für ein Umsetzen von verschiedenen Impulsen im Heimatland. Beispielsweise erzählt die indische Freiwillige, eine studierte Architektin, dass sie gerne eine Nichtregierungsorganisation (NGO) gründen möchte und den Freiwilligendienst als Erfahrungsgewinn in diese Richtung betrachtet:

And also because I had decided I would re-schedule in the direction of an NGO or something and I had never worked in an NGO. Like, I know how an architecture firm works, but the structure of an NGO [...], I had no idea. And, I knew I didn't want to do architecture, I didn't want to build things. So I thought: Okay, it's maybe time I take a different direction.

Andere Interviewte erzählen von Ideen für Kursen oder Workshops, die sie nach dem Jahr umsetzen möchten. Die Beispiele zeigen, dass Wissen und Erfahrungen weitergegeben werden. Es kann argumentiert werden, dass die Interviewten ihr persönliches - und an manchen Stellen auch fachliches - Empowerment in ihrer Heimat umsetzen. 
Andererseits kritisiert bspw. ein tansanischer Freiwilliger und ausgebildeter Jurist, dass ihn der Dienst fachlich nicht weitergebracht habe. Diese Beobachtungen decken sich mit dem Weltwärts-Evaluationsbericht (Scheller/Krämer/Hielscher 2017: ix): In gut angepassten Einsatzstellen könnten die Freiwilligen ihre Fähigkeiten in Deutschland besser einbringen, während des Dienstes fachlich mehr lernen und in der Heimat anwenden. Da das Programm jedoch im Rahmen des Bundesfreiwilligendienstes (BFD) explizit auf unterstützende Hilfstätigkeiten ausgelegt ist, ist dies im Moment schwerlich möglich. Weltwärts versteht sich als Lerndienst - dieser könnte bei einer offeneren Handhabe der Einsatzplatzvergabe größer ausfallen. Ein Ändern der Rahmenbedingungen kann hier entgegenwirken. Aufgabe der Aufnahmeorganisation sollte es sein, Einsatzstellen zu finden, die im Rahmen des BFD möglichst gut auf die Interessen und Möglichkeiten der einzelnen Freiwilligen passen.

Während des Jahres sind Konfrontationen mit rassistischen Denkweisen in verschiedensten Ausprägungen (über tatsächliche Anfeindungen und über rassistische Kommentare, bis hin zu positivem Rassismus) normale Erlebnisse in einem Süd-Nord-Freiwilligendienst. Exemplarisch zu stereotypen Denken in Deutschland sind die Aussagen einer Freiwilligen aus Togo. Sie stellt die Frage, warum in ihrem Heimatland so viele positive Dinge über Europa vermittelt werden. Dort gäbe es „einen [ideellen] Platz für Europäer“. In Togo würden positive Bilder über den Norden hervorgerufen. In Deutschland jedoch begegnet ihr das genaue Gegenteil: Afrika wird verbunden mit Armut, Tieren und Hunger - negativen Assoziationen. Dies hat sie verunsichert und sie stellt sich nun die Frage, ob Europa nicht in ihrer Heimat zu sehr glorifiziert wird. ${ }^{1}$ Der Schluss, den die togoische Freiwillige für sich gezogen hat ist, so viel wie möglich über Togo und Afrika zu erzählen und so das Bild der Menschen zu ändern: „Sie müssen wissen, dass Afrika auch viele Vorteile hat. Und jetzt fühle ich mich besser." Ihre Vorträge und Erzählungen zu „meinem Afrika, meinem Togo“ sind eine Strategie, ihrem persönlichen Frust über die Rassismen der Menschen in Deutschland zu begegnen.

Das Beispiel zeigt, wie sehr das Denken des „defizitären Anderen“, des „Othering“ (Ziai 2016: 403) auf deutscher Seite internalisiert ist. Das Freiwilligenprogramm könnte jedoch ein Weg sein, diese Vorurteile langsam abzubauen.

Ein weiteres Ergebnis der Interviews war der Abbau von hierarchisierendem Denken innerhalb bzw. zwischen den Ländern und Kontinenten der Teilnehmenden. Von besonderem Interesse sind dabei die Aussagen einer Freiwilligen aus Sri

1 Der Globale Süden als diskursives Gegenbild des Globalen Nordens und das „Glorifizieren Europas“ zeigt sich auch in Erwartungshaltungen von Familien und Freund_innen in der Heimat, die bspw. teure Geschenke und Mitbringsel fordern. Dies wird mehrfach in den Interviews angedeutet. 
Lanka, die sehr offen ihre eigenen Sichtweisen und die Vorstellungen ihres Umfeldes zum afrikanischen Kontinent kommuniziert:

Because, mostly in an Asian country you get to know an African person only through television [...]. And it is not only Europeans who see Africans in a different perception like: "Their country is very dry. Or: They are very poor. They eat, like, animals or, like, people." Something like a misconception. Because, I personally had that misconception 'till I met my friends in Africa. [...] But, to be honest, when I first met my African friends in the first seminar of my volunteer service, actually I was scared of them. I was really, really scared to see them. Maybe, because of the perception I had. The influence I had in my life with the television. Because, they are always with a bow and arrow.[...] The things I saw is that they are always dirty, with mud and dusk and poor houses ... And they don't have good food and they don't have proper clothing or proper education. I felt like they are also a part of an animal kingdom, they are not human beings. Actually, I felt that they are not humans. So, I had a total misconception!

Demnach wird Afrika gleichgesetzt mit Natur bzw. Primitivität (die Freiwillige spricht in diesem Kontext von „,animal kingdom“), dem Jagen mit Pfeil und Bogen Dreck und Armut; eine Region ohne „,proper education“. Die Aussagen, die sie tätigt, entsprechen dem Bild, das auch in Deutschland häufig von Afrika entworfen wird. Dabei wird eine europäisch ausgestaltete (wie auch immer geartete) Norm gesetzt, anhand derer alles andere als negativ gewertet wird. Der Dualismus stellt westeuropäisches Denken als Kultur, Errungenschaft und Norm der Gestaltung von Gesellschaften dar. Diese Stereotype, nämlich die „Verschmelzung mit der Natur“ und die daraus folgende Kulturlosigkeit des Anderen sind „Zeugnis gängiger ethnozentristisch-rassistischer Konstruktionen und Einstufungen“ (Arndt 2004: 100). Dies wiederum impliziert einen Eurozentrismus, wie er bspw. von Said (1978), Chakrabarty (2002: 283) oder Sutterlüty (2002: 13 ff) kritisiert wird. Interessant ist jedoch, dass die Freiwillige selbst aus einer ehemaligen Kolonie (Sri Lanka) stammt. Eine hierarchisierende Weltordnung ist dennoch ein von ihr selbst internalisiertes Denken. Ihrer Logik folgend steht Afrika „unter“ Asien, denn in ihren Aussagen impliziert sie, dass all die negativen Dinge aus Afrika in Asien (bzw. Sri Lanka) nicht anzutreffen sind. Gleichzeitig stellt sie aber auch klar, dass Europa an höchster Stelle steht, „because it's like a rich country“2. Die Freiwillige versucht, die an sich selbst beobachteten rassistischen Denkweisen mit einem Verweis auf mediale Berichterstattung (insbesondere Dokumentationen in der BBC) zu erklären. Positiv kann hervorgehoben werden, dass sie ihr Bild über Afrika und Afrikaner_innen anscheinend aufgrund des persönlichen Kontaktes geändert hat. Der Austausch mit anderen jungen Menschen aus aller Welt trägt auch dazu bei, persönliche Einstellungen zu ändern. Augenscheinlich

2 "We all know that Europeans how they are, because, it's always like a rich country." 
müssen nicht nur die Impulse der Freiwilligen innerhalb ihres Umfelds in Deutschland und im Heimatland, sondern auch innerhalb der Freiwilligengruppen fokussiert werden. Internationaler Austausch und Kontakte sowie gemeinsames Lernen sind zentrale Punkte, um Dialoge untereinander zu öffnen (Mischke 2010; SchneiderWohlfahrt 2013).

\subsubsection{Helfen?! Spenden?!}

Da die Interviewsituation bewusst offen gehalten wurde, entwickelten sich nicht alle Gespräche in dieselbe Richtung. Zwei Interviewte sprachen von sich das Thema „Helfen und Spenden“ an. Eine ruandische Freiwillige berichtet, wie in ihrer Heimat die Menschen keine Erfahrungen mit den Abläufen zum Erhalt von Spenden haben. Ferner, sagt sie, würde mit den Hilfszahlungen fast schon gerechnet:

We are not really informed, but what we know is that the church in Germany supports everything. And we get some money from Germany when we have to construct something. [...] But, the members [in Rwanda] are still not knowing what happened. They think: 'The church in Germany will give us the money.' Something like that. But we don't know really how or about the procedures and who did it or how the cooperation works.

Im Gegensatz dazu legt ein tansanischer Freiwilliger in seiner Erzählung den Fokus auf die Idee des „Helfens“ in Geberländern, wo Spender_innen über stereotype Werbung und Inhalte zum Geben aufgefordert werden. ${ }^{3}$ Diese führten dazu, dass sich - auf Geberseite - die Bilder des defizitären Anderen manifestieren. Gleichzeitig würden Projekte unterstützt, die nicht unbedingt zielführend seien.

Die Stereotypisierungen, von denen die Freiwilligen - insbesondere diejenigen aus afrikanischen Ländern - berichten, sind ein Teil der einseitigen Darstellung des Kontinents in deutschen Medien, aber auch in der Werbung. Beiträge von Kiesel und Bendix (2016) oder glokal e. V. und Initiative Schwarze Menschen in Deutschland e. V. (2017) kritisieren die Reproduktion von Stereotypen in Spendenwerbungen. Sie sei ein wichtiges Puzzlestück der gedanklichen Verbindung von Armut, Hilfsbedürftigkeit und Rückständigkeit mit dem afrikanischen Kontinent. Schwarze und People of Color werden in der Öffentlichkeit anders dargestellt als Weiße und so auf einen Aspekt reduziert (Kiesel/Bendix 2016: 484). Der tansanische Freiwillige spricht von einem Impuls des „Helfens“ oder „Gutes tun“. Dies

3 "We had this meeting last time and some of the leaders [of mission organizations] was that they find it that most people feel important when they make a contribution to something that is not working. And therefore, they feel important to something that wouldn't have happened if they didn't contribute. And to do that, they have to be convinced by inputs or by naming things in a way could be stereotypical. But, I think that there has to be a dialogue, or there has to be a movement that teaches people that that's not really the point of helping. The point of helping is to change something somewhere in the world if they want help. And maybe it's better to empower than just support." 
mag ehrenhaft erscheinen, steht aber dennoch in einer kolonialen Tradition. Hier kommt eine Dichotomisierung zwischen „uns“ und „den anderen“ zum Tragen (Miles 1991: 117). „Wir“ „helfen“ „den Hilfsbedürftigen“. Ob die Spenden sinnvoll sind, steht auf einem anderen Blatt. Der Freiwillige, der dieser Analogie folgend ein „Anderer“ ist, ist irritiert. Festzuhalten ist, dass Bilder und Spendenwerbung eine Wirkmacht haben, die die Freiwilligen (und andere Menschen, die vermeintlich nicht „deutsch“ aussehen) zu spüren bekommen.

Auf der anderen Seite stehen die von der oben zitierten ruandischen Freiwilligen dargestellten Beispiele: Sie gibt an, dass die Mitglieder ihrer Gemeinde in der Regel nicht wüssten, wie Gelder, mit deren Hilfe große Bauprojekte realisiert werden, sie erreichten. Einzig, dass sie aus Deutschland kommen, sei klar. Einige Pastor_innen oder andere Eliten seien diejenigen, die mit der Spendenbeschaffung vertraut sind. $\mathrm{Ob}$ dies Absicht ist oder nicht, bleibt offen. Was in der Bevölkerung jedoch ankommt, ist, dass die Kirche in Deutschland „alles unterstütze“.

Die Aussagen zeigen die Ambivalenz von Spenden. Es mag solidarisch sein, sich gegenseitig zu unterstützen, aber es generiert auch Abhängigkeiten. Das von der ruandischen Interviewten geschilderte Dilemma ist exemplarisch für verschiedene Regionen der Welt, in der die Wirtschaft längst abhängig von Hilfszahlungen geworden ist. Auch wenn das im Interview gegebene Beispiel der gebauten Kirche sicher nicht ökonomische Entwicklung zum primären Ziel hatte, so ist es dennoch ein Mosaikstein der allgegenwärtigen Hilfs- bzw. „Entwicklungsindustrie“ (Korf/ Rothfuß 2016: 178). Auch im kirchlichen Kontext stellt bspw. Kileo (2014) fest, dass „die Kirchen des Globalen Südens die Augenhöhe nicht erreichen können, solange die ,Macht des Geldes' weiterhin durch die Wohltätigkeit des Nordens so starken Einfluss hat, dass sie sich als Dominierungsstrategie auswirkt“ (Kileo 2014: 270). Das letzte Wort darüber, was finanziert wird (und in welcher Höhe), liegt letztlich in Deutschland. Hier kann die Agenda dessen, was zu beachten ist, gesetzt werden. Die Normen und Werte Deutschlands müssen dabei beachtet werden.

„Maybe, it is better to empower than to support“, sagt der tansanische Freiwillige zum Abschluss. Dieser Satz fasst eine postkolonial sensible Sicht auf die Aspekte von „Helfen?!“ und „Spenden?!“ gut zusammen: Die Tat des Helfens führt leicht zu einer Abhängigkeit des Geholfenem gegenüber dem Helfenden - vor allem, wenn dies an finanzielle Leistungen gekoppelt ist. Empowerment hingegen führt unter anderem dazu, Machtstrukturen zu hinterfragen und in eine Position gehoben zu werden, selbst Verantwortung zu übernehmen und Entscheidungen zu treffen. Der ständigen - bewusste oder unbewusste - Reproduktion der Superiorität des Globalen Nordens und der Inferiorität des Globalen Südens kann durch Ermächtigungsstrategien entgegengewirkt werden. Die eingangs zitierten Macht- und Abhängigkeitsverhältnisse innerhalb der Entwicklungszusammenarbeit und ihrer Spendenakquise sind dennoch weiterhin nachzuweisen. 


\section{Fazit}

Der vorliegende Beitrag stellte die Ansichten und Beobachtungen von Weltwärts Süd-Nord-Freiwilligen in den Mittelpunkt der Analyse. Aufgrund des bisher in der Literatur wenig beachteten Themas der Entwicklung in der Süd-Nord-Komponente, wurde dieses als Untersuchungsgegenstand gewählt. In den Antworten der Freiwilligen zur Frage der Entwicklungszusammenarbeit wurde versucht, ihr Verständnis von Entwicklung zu rekonstruieren.

In der Analyse wurde herausgearbeitet, dass die Befragten drei Dimensionen von „Entwicklung“ in ihrem Dienst sehen, nämlich (1) ihre persönliche Entwicklung, (2) politische, soziale und manchmal auch ökonomische Entwicklungen in Deutschland und in der Heimat sowie (3) die Frage der Entwicklungshilfe.

Persönliche Entwicklung findet den Ergebnissen nach zunächst auf der individuellen Ebene statt: "Personally, I have grown strong", sagt die interviewte Inderin. Weltwärts ist als Lerndienst konzeptualisiert (Weltwärts o. J.). Das persönliche Wachsen ist ein Prozess, der in fast allen Interviews geäußert wird und sich auch mit den Erkenntnissen anderer Studien deckt (Schneider 2016).

Der Dienst berührt aber auch die Leben der Menschen in Deutschland und das persönlichen Umfelds in der Heimat. Hervorzuheben ist der Abbau von Stereotypen. So berichtet eine Freiwillige aus Togo, dass sie versuche das einseitige Afrika-Bild in Deutschland durch ein systematisches Erzählen von „ihrem Afrika, ihrem Togo“ zu verändern. Weiterhin hilft der Dienst, Ängste und Stereotype innerhalb der Freiwilligengruppe abzubauen. Die ehrliche Aussprache einer Sri Lankischen Freiwilligen über anfänglichen Ängste gegenüber den afrikanischen Freiwilligen ist hier ein gutes Beispiel.

Gleichzeitig erläutern die Befragten auch, dass Erfahrungen und neu erlernte Dinge in der Heimat weitergegeben, angewandt und somit politische, soziale und unter Umständen auch ökonomische Entwicklungen generiert werden. Es gibt also eine vielschichtige Multiplikation von Erfahrungen (vgl. z. B. Richter und Haas 2019: 24).

Neben dieser persönlichen Dimension wurde in den Interviewsituationen auch die strukturelle bzw. politische Dimension von Entwicklung angesprochen. Dabei äußerte sich ein tansanischer Freiwilliger zu der deutschen Sicht auf Spenden, in deren Werbung Stereotype des armen Globalen Südens reproduziert würden (vgl. auch Initiative Schwarze Menschen in Deutschland e.V. 2017; Kiesel und Bendix 2016; glokal e.V.). Auf der anderen Seite ging eine ruandische Freiwillige darauf ein, wie in ihrer Heimat wenig Wissen über Abläufe zum Erhalt von Spenden herrschen würde. „Maybe, it is better to empower than to support“, sagt der tansanische Interviewte zum Abschluss. Im Idealfall tut der Freiwilligendienst Weltwärts dies: die Freiwilligen ermächtigen, selbst Dinge in Bewegung zu setzen und im Idealfall multiplikatorisch zu wirken. „There is more confidence in terms of how I could create social change", sagt eine Interviewte über sich. 
Letztlich zeigt sich in den Interviews, wie vielfältig der entwicklungspolitische Ansatz des Weltwärts-Programmes ist. So geht es allenfalls sekundär um einen Anstieg des Pro-Kopf-Einkommens, sondern vielmehr um die Bildung von zwischenmenschlichen Beziehungen und Ermächtigung, sowie non-formalen Lernerfahrungen der Freiwilligen. Outcomes sind Achtung voreinander, sowie Respekt und Toleranz. Abschließend kann resümiert werden, dass die interviewten Freiwilligen ein mehrdimensionales Bild von Entwicklung zeichnen und es als ganzheitliches Konzept ansehen. Es geht darum, sich persönlich weiterzuentwickeln, Dinge kritisch zu hinterfragen und als Multiplikator_innen die verschiedenen Denkweisen und Ansichten weiterzugeben. Die Impulse führen zu mehrschichtigen Formen der Entwicklung. Sie kann ökonomischer Art sein, umfasst aber ebenso ein Umdenken, Horizonterweiterung und gesellschaftliche Transformationsprozesse - sowohl in den Heimatländern als auch in Deutschland.

\section{Literaturverzeichnis}

Arndt, Susan (2004): Afrika und die deutsche Sprache. Ein kritisches Nachschlagewerk, Münster.

Eriksson Baaz, M. (2005). The paternalism of partnership: A postcolonial reading of identity in development aid, London.

Bhabha, Homi K. (1994): The Location of Culture, London.

Blatter, Joachim, Phil C. Langer und Claudius Wagemann (2017): Qualitative Methoden in der Politikwissenschaft. Eine Einführung, Wiesbaden.

Conrad, Sebastian (2012): Kolonialismus und Postkolonialismus: Schlüsselbegriffe der aktuellen Debatte, in: Aus Politik und Zeitgeschichte, Jg. 62, Heft 44-45, S. 3-9.

Chakrabarty, Dipesh (2002): Europa provinzialisieren. Postkolonialität und die Kritik der Geschichte, in: Jenseits des Eurozentrismus. Postkolonial Perspektiven in den Geschichtsund Kulturwissenschaften, hrsg. von Conrad, Sebastian und Randeria, Shalini, Franfurt a. M., S. 283-312.

Castro Varela, do Mar Maria und Nikita Dhawan (2015): Postkoloniale Theorie. Eine kritische Einführung. 2. Aufl., Bielefeld.

Eckert, Andreas (2006): Kolonialismus, Frankfurt a. M.

Escobar, Arturo (1995): Encountering development: the making and unmaking of the third world, Princeton.

Flick, Uwe, Ernst von Kardoff und Ines Steinke (Hrsg.) (2007): Qualitative Sozialforschung. Ein Handbuch, Reinbeck.

glokal e. V. (2016): Das Märchen von der Augenhöhe. Macht und Solidarität in Nord-SüdPartnerschaften, Berlin,

glokal e. V. und Initiative Schwarze Menschen in Deutschland e. V. (Hrsg.): Die Spitze des Eisbergs. Spendenwerbung der internationalen Hilfsorganisationen - Kritik und Alternativen, Berlin.

Hall, Stuart (1994): Rassismus und kulturelle Identität, Hamburg. 
Heinze, Thomas (2001): Qualitative Sozialforschung. Einführung, Methodologie und Forschungspraxis, München.

Kerner, Ina (2012): Postkoloniale Theorien. Zur Einführung, Hamburg.

Kiesel, Timo und Daniel Bendix (2016): White Charity: Eine postkoloniale, rassismuskritische Analyse der entwicklungspolitischen Plakatwerbung in Deutschland, in: Peripherie Politik Ökonomie Kultur, Jg. 30, Heft 120, S. 482-494.

Kileo, Emmanuel (2014): Weiß-Sein als ideologisches Konstrukt in kirchlichen Süd-Nord Partnerschaften, Neuendettelsau.

Kontzi, Kristina (2011). Ich helfe, du hilfst, ... ihnen wird geholfen. Der Freiwilligendienst Weltwärts reproduziert altbekannte Strukturen, in: iz3w, Heft 323, S. 40-42.

Kontzi, Kristina (2015): Postkoloniale Perspektiven auf „Weltwärts“,Baden-Baden.

Korf, Benedikt und Eberhard Rothfuß (2016): Nach der Entwicklungsgeographie, in: Humangeographie kompakt, S. 163-183.

Mayring, Philipp (2002): Einführung in die qualitative Sozialforschung, Weinheim-Basel.

Mayring, Philipp (2015): Qualitative Inhaltsanalyse. Grundlagen und Techniken. 12. Aufl., Wienheim-Basel.

Miles, Robert (1991): Rassismus. Einführung in die Geschichte und Theorie eines Begriffs, Hamburg.

Mischke, Jakob (2010): Grenzüberschreitende Kooperation und zivilgesellschaftliche Kontakte, in: Серія “Політичні науки”, S. 234-238.

Müller, Franziska und Aram Ziai (2015): Eurozentrismus in der Entwicklungszusammenarbeit, in: Aus Politik und Zeitgeschichte, Jg. 65, Heft 7-9, S. 8-15.

Polak, Jan Tobias, Kerstin Guffler und Laura Scheinert (2017): Weltwärts-Freiwillige und ihr Engagement in Deutschland, Bonn.

Reichertz, Jo (2014): Empirische Sozialforschung und soziologische Theorie, in: Handbuch Methoden der empirischen Sozialforschung, hrsg. von Nina Baur und Jörg Blasius,Wiesbaden, S. 65-80.

Repenning, Alexander (2016): Warum machen sie da(s) mit? Motivationen und Sichtweisen von Weltwärts-Partnerorganisationen im Globalen Süden - Eine explorative Studie in Lima, Peru, in: Voluntaris, Jg. 4, Heft 1, S. 46-67.

Said, Edward (1978): Orientalism, New York.

Scheller, Jan Oliver, Matías, Krämer und Hanna Hielscher (2017): Evaluierungsbericht der Weltwärts-Süd-Nord-Komponente, Bd. I Hauptbericht, hrsg. vom Bundesministerium für Wirtschaftliche Zusammenarbeit und Entwicklung, Bonn.

Schneider, Marie-Luise (2016): „Ich wollte diesen persönlichen Wachstumsschritt machen“ Eine ethnologische Untersuchung zur Motivation von Incoming-Freiwilligen in Deutschland, in: Voluntaris, Jg. 4, Heft 2, S. 192-213.

Schneider-Wohlfahrt, Ursula (2013): Fremdheit überwinden. Theorie und Praxis des interkulturellen Lernens in der Erwachsenenbildung, Berlin.

Skoruppa, Daniel (2016): Weltwärts Süd-Nord - Freiwilligenaustausch auf Augenhöhe? Eine machtkritische Auseinandersetzung mit dem entwicklungspolitischen Freiwilligendienst, Erfurt. 
Sutterlüty, Beate (2002): Jenseits des Eurozentrismus: postkoloniale Perspektiven in den Geschichts-und Kulturwissenschaften, Frankfurt a. M..

Spivak, Gayatri Chakravorty (1988): Can the subaltern speak?, in: Marxism and the Interpretation of Culture, hrsg. von Cary Nelson und Lawrence Grossberg, University of Illinois Press, S. 21-78.

Weltwärts (o. J.): Programm. Der entwicklungspolitische Freiwilligendienst stellt sich vor, www.weltwaerts.de/de/programm.html (09.04.2019).

Ziai, Aram (2016): Postkoloniale Perspektiven auf „Entwicklung “, in: Peripherie - Politik Ökonomie Kultur, 30. Jg., Heft 120, S. 399-426. 